\title{
Three dimensional magnetic field reconstruction of artificial Skyrmion heterostructures
}

\author{
Sheng Zhang ${ }^{1}$, Amanda Petford-Long ${ }^{1}$, Charudatta Phatak ${ }^{1}$ \\ ${ }^{1}$ Materials Science Division, Argonne National Laboratory, Lemont, IL 60439, USA
}

The Skyrmion spin structure is a topologically stable state in which the spins point in all directions wrapping around a sphere [1]. The Skyrmion configuration is not only important from a fundamental aspect in condensed matter physics as it can produce unconventional spin-electronic phenomena [2] but also from a technological point of view as a spintronic material [3]. In bulk crystals and thin films, the Skyrmion spin texture arises from the helical spin structure and is stabilized by the DzyaloshinskiiMoriya (DM) interaction. Lorentz microscopy has been previously used to study the real-space configuration of Skyrmions in thin films of $\mathrm{Fe}_{0.5} \mathrm{Co}_{0.5} \mathrm{Si}$, and $\mathrm{FeGe}$. However, since the magnetization of a Skyrmion state rotates in a continuous manner from one out-of-plane direction to the opposite one, a careful analysis of this state requires a complete three-dimensional analysis of magnetization. In this work, we will present the results from vector field electron tomography combined with Lorentz transmission electron microscopy (LTEM) to study such three-dimensional magnetization.

The phase change $\varphi_{e}+\varphi_{m}$ of an electron wave traveling through a thin foil can be expressed in terms of tomographic quantities; the electrostatic phase shift $\varphi_{e}$ corresponds to the scalar x-ray transform of the electrostatic potential, whereas the magnetic phase shift $\varphi_{m}$ is described by the vector X-ray transform of the magnetic vector potential [4]. The magnetic phase shift from the sample can be reconstructed using either off-axis or in-line holography. In this work, the tilt series was performed along two orthogonal directions. The recovered phase shift was then used to reconstruct the three-dimensional components of magnetization using standard iterative methods such as SIRT. We will discuss the reconstruction results and improvements achieved by using the iterative reconstruction methods.

In our previous work on magnetic heterostructures, we have successfully shown that by controlling the macroscopic energy terms of magnetic layers, it is possible to stabilize novel topological states at room temperatures such as a "meron" state [5]. We will report on "artificial" Skyrmion lattices consisting of $\mathrm{Co}$ and $\mathrm{Co} / \mathrm{Pt}$ multilayers that can be stabilized at room temperature. As a result, instead of having chiral DM interactions, the spin texture is controlled by the dipole-dipole interactions (magnetostatic interactions) and interlayer exchange interactions, which can lead to spin canting to create artificial Skyrmion spin structure. We have fabricated heterostructures consisting of $\left[\mathrm{Co}_{0.3} / \mathrm{Pt}_{1}\right]_{8}$ multilayers that have perpendicular anisotropy and thin layer of Co $(10 \mathrm{~nm})$ on top that has in-plane anisotropy as shown in Fig. 1(a). They were then patterned into disc structures using focused ion beam milling to introduce shape anisotropy to form vortex structures in the Co layer. Simulations were performed to understand the image contrast arising from the various layers in the heterostructures. Fig. 1(b)-(d) show the magnetization maps used to simulate the LTEM images, and Fig. 2(a)-(c) show the simulated underfocus images of the disc at $-40^{\circ}, 0^{\circ}$, and $+40^{\circ}$ tilt respectively. Fig. 2(d)-(f) show the experimentally obtained underfocus images at various tilt angles as indicated. In both simulated and experimental set of images, the domain contrast arising from the the out-of-plane magnetization can only be seen in the tilted images. It can also be seen that the additional contrast due to out-of-plane component changes contrast from black to white and vice-versa when tilted from $-40^{\circ}$ to $+40^{\circ}$ as 
indicated by the arrows in Fig. 2. We will report on the 3D magnetic field obtained from these discs and discuss the implication towards creationg of Skyrmion states.

\section{References}

[1] T. H. R. Skyrme, Proc. R. Soc. A, 262, 237 (1961).

[2] Y. Onose, N. Takeshita, C. Terakura, H. Takagi, and Y. Tokura, Phys. Rev. B, 72, 224431 (2005).

[3] X. Z. Yu et. al., Nat. Commun., 3, 988 (2012).

[4] C. Phatak, M. Beleggia, and M. De Graef, Ultramicroscopy, 108, 503 (2008).

[5] C. Phatak, A. Petford-Long, O. Heinonen, Phys. Rev. Lett., 108, 067205 (2012).

[6] This work was supported by U.S. Department of Energy (DOE), Office of Science, Materials Sciences and Engineering Division.

(a)

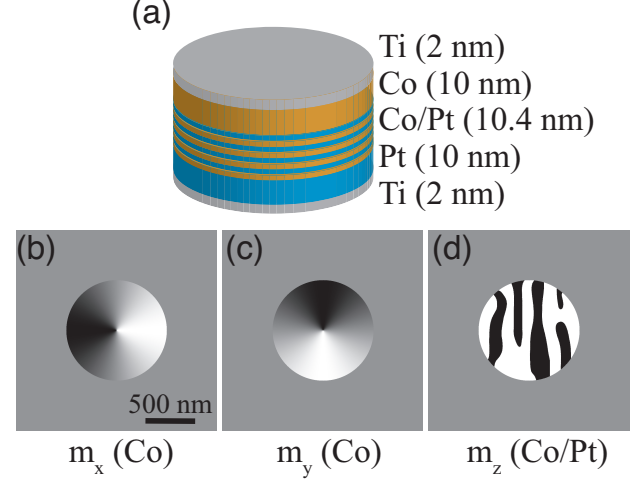

Figure 1: (a) Schematic showing the various layers in the magnetic heterostructure. (b)-(d) shows the grayscale representation of the magnetization maps for the $\mathrm{Co}$ and $\mathrm{Co} / \mathrm{Pt}$ layer.

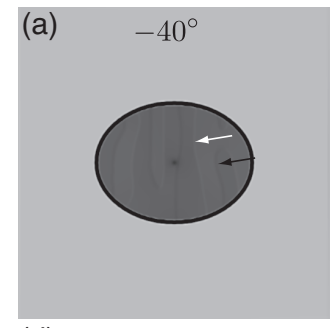

(d)

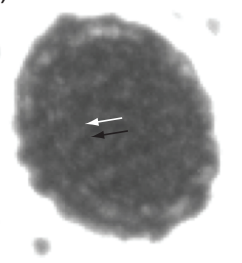

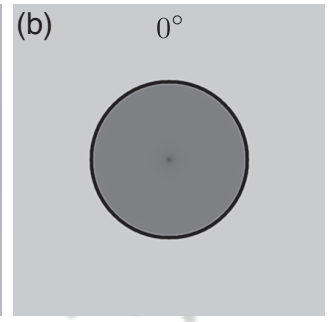

(e)

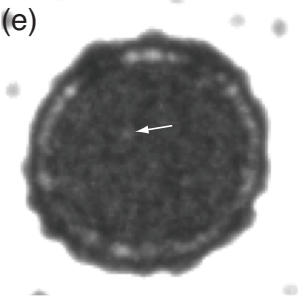

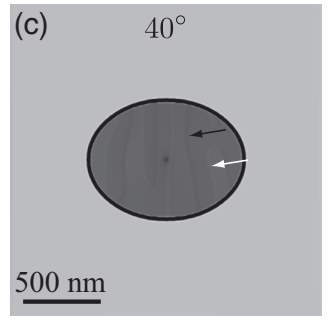

(f)

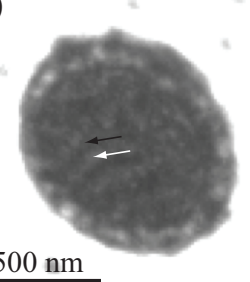

Figure 2: (a)-(c) Simulated underfocus images showing the contrast from the vortex in the Co layer and additional domain contrast from the Co/Pt layers under tilted conditions, (d)-(f) Experimentally obtained underfocus images showing the domain contrast under varying tilting conditions. 